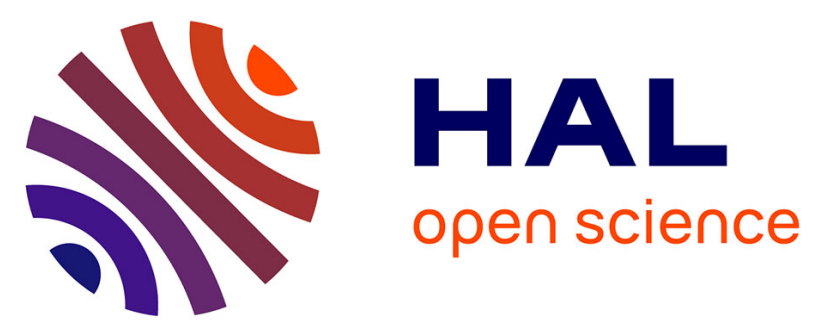

\title{
Validation of Finite Element Image Registration-based Cardiac Strain Estimation from Magnetic Resonance Images
}

Ezgi Berberoglu, Christian T Stoeck, Philippe Moireau, Sebastian Kozerke, Martin Genet

\section{To cite this version:}

Ezgi Berberoglu, Christian T Stoeck, Philippe Moireau, Sebastian Kozerke, Martin Genet. Validation of Finite Element Image Registration-based Cardiac Strain Estimation from Magnetic Resonance Images. GAMM 2019 - 90th Annual Meeting of the International Association of Applied Mathematics and Mechanics, Feb 2019, Vienna, Austria. 10.1002/pamm.201900418 . hal-02375735

\section{HAL Id: hal-02375735 \\ https://hal.science/hal-02375735}

Submitted on 25 Nov 2019

HAL is a multi-disciplinary open access archive for the deposit and dissemination of scientific research documents, whether they are published or not. The documents may come from teaching and research institutions in France or abroad, or from public or private research centers.
L'archive ouverte pluridisciplinaire $\mathbf{H A L}$, est destinée au dépôt et à la diffusion de documents scientifiques de niveau recherche, publiés ou non, émanant des établissements d'enseignement et de recherche français ou étrangers, des laboratoires publics ou privés. 


\title{
Validation of Finite Element Image Registration-based Cardiac Strain Estimation from Magnetic Resonance Images
}

\author{
Ezgi Berberoğlu ${ }^{1,2,3 *}$, Christian Stoeck $^{1}$, Philippe Moireau ${ }^{3,2}$, Sebastian Kozerke $^{1}$, and Martin Genet ${ }^{2,3,1}$ \\ ${ }^{1}$ Institute for Biomedical Engineering, University and ETH Zurich, Gloriastrasse 35, 8092 Zurich, Switzerland \\ ${ }^{2}$ Laboratoire de Mécanique des Solides, École Polytechnique/CNRS/Université Paris-Saclay, Palaiseau, France \\ ${ }^{3}$ M $\Xi D I S I M$ team, Inria/Université Paris-Saclay, Palaiseau, France
}

\begin{abstract}
Accurate assessment of regional and global function of the heart is an important readout for the diagnosis and routine evaluation of cardiac patients. Indeed, recent clinical and experimental studies suggest that compared to global metrics, regional measures of function could allow for more accurate diagnosis and early intervention for many cardiac diseases. Although global strain measures derived from tagged magnetic resonance (MR) imaging have been shown to be reproducible for the majority of image registration techniques, the measurement of regional heterogeneity of strain is less robust. Moreover, radial strain is underestimated with the current techniques even globally. Finite element (FE)-based techniques offer a mechanistic approach for the regularization of the ill-posed registration problem. This paper presents the validation of a recently proposed FE-based image registration method with mechanical regularization named equilibrated warping. For this purpose, synthetic 3D-tagged MR images are generated from a reference biomechanical model of the left ventricle (LV). The performance of the registration algorithm is consequently tested on the images with different signal-to-noise ratios (SNRs), revealing the robustness of the method.
\end{abstract}

Copyright line will be provided by the publisher

\section{Introduction}

Assessment of regional myocardial function is important for the diagnosis of the majority of cardiovascular diseases [1]. Conventional techniques used to quantify cardiac function are widely performed based on global measures that may result in the misinterpretation of cardiac performance due to the lack of sensitivity to regional variability $[2,3]$. Moreover, changes in these metrics are generally observed in the late stages of the diseases for most of the cases [4]. Among all imaging methods, cardiac magnetic resonance (CMR) tagging has been developed for regional heart motion analysis, allowing to superimpose tag line patterns onto the heart to assess ventricular wall deformation [5]. Studies on the healthy human heart using CMR tagging report regional differences in strain distribution [6,7]. Although this technique is shown to be promising for the assessment of regional deformation, motion quantification techniques are highly affected by the two main error sources, coarse resolution and low SNR, commonly observed in in vivo tagged CMR images. Decrease in image resolution due to the saturation bands generated in the tagged images introduces systematic error due to partial voluming at edges $[8,9]$. Moreover, change in SNR causes random error which is solved by various regularization techniques implemented in the motion tracking algorithms. FE-based image registration techniques have been applied to different imaging modalities for the quantification of motion distribution in the heart [10]. Different from other methods, FE models offer a mechanics-based approach for stress/strain prediction while accounting for the structural properties of the LV myocardium. These models require prescription of appropriate boundary conditions and a sound material model to characterize the cardiac constitutive behavior.

The objective of the present work is to validate a recently proposed FE-based image registration method with mechanical regularization, named equilibrated warping [11], for strain quantification from 3D tagged images. For this purpose, we generate synthetic 3D tagged images from a reference biomechanical model of LV [12] that represents ground truth. The registration algorithm is then run on images with different noise levels. The computed displacement fields and associated strain components are compared to the ground truth from the reference biomechanical model to demonstrate the robustness of the proposed registration technique with respect to image noise.

\section{Methods}

Formulation of FEM-Based Image registration. A continuum formulation of the image registration problem is introduced and later discretized through the FE method. Considering the reference and current configurations of the object represented by the images, $\Omega_{0}$ and $\Omega_{t}$, related image intensity fields are denoted by $I_{0}$ and $I_{t}$. A nonlinear deformation map $\varphi(\mathbf{X})$ is described between these two configurations to map the reference points $\mathbf{X} \in \Omega_{0}$ onto their spatial counterparts $\mathbf{X}=\varphi(\mathbf{X})=$ $\mathbf{X}+\mathbf{U}(\mathbf{X}) \in \Omega_{t}$, where $\mathbf{U}$ is the displacement field. In the presence of image noise, the problem is no longer compatible with mechanics and requires a regularization of the displacement field. Hence, the problem of finding the displacement field

* Corresponding author: e-mail berberoglu@biomed.ee.ethz.ch 
can be formulated as

$$
\text { find } \mathbf{U}=\underset{\left\{\boldsymbol{U}^{*}\right\}}{\operatorname{argmin}}=\left\{J\left(\boldsymbol{U}^{*}\right)=(1-\beta) \Psi^{\mathrm{im}}\left(\boldsymbol{U}^{*}\right)+\beta \Psi^{\mathrm{reg}}\left(\boldsymbol{U}^{*}\right)\right\},
$$

which aims to minimize the functional $J$ expressed in terms of $\beta$, regularization strength, $\Psi^{\mathrm{im}}$, image similarity metric, and $\Psi^{\text {reg }}$, regularization energy

$$
\begin{aligned}
& \Psi^{\mathrm{im}}(\boldsymbol{U})=\frac{1}{2}\left\|I_{t} \circ \boldsymbol{\varphi}-I_{0}\right\|_{L^{2}\left(\Omega_{0}\right)}^{2}, \\
& \Psi^{\mathrm{reg}}(\boldsymbol{U})=\sum_{K} \frac{1}{2}\|\mathbf{D i v}(\mathbf{F} \cdot \mathbf{S})\|_{L^{2}(K)}^{2}+\sum_{F} \frac{1}{2 h} \llbracket(\mathbf{F} \cdot \mathbf{S} \cdot \mathbf{N}) \rrbracket_{L^{2}(F)}^{2},
\end{aligned}
$$

where $\mathrm{K}, \mathrm{F}$ and $\mathbf{N}$ represent the set of finite elements, the set of interior facets, and the facets normal, respectively, $\mathbf{S}$ and $\mathbf{F}$ are the second Piola-Kirchhoff stress tensor and transformation gradients, respectively, and $\mathrm{h}$ is a characteristic length of the elements. See [11] for more details. The novelty in the method is the regularization technique which is a continuum finite strain formulation of the equilibrium gap principle introduced in [13]. Compared to other regularization techniques, equilibrated warping brings a mechanics-based insight, satisfying the basic mechanical balance principles to regularize the problem. In addition to the equations specified above, Equation (3) requires the specification of constitutive model, chosen here as a Ciarlet-Geymonat compressible hyperelastic potential [14]

$$
\rho_{0} \psi=\frac{\kappa}{2}\left(J^{2}-1-\ln (J)\right)+\frac{\mu}{2}\left(I_{C}-3-2 \ln (J)\right),
$$

in terms of bulk and shear modulus, $\kappa$ and $\mu$, volume map, $J=\operatorname{det}(\mathbf{F})$, and $I_{C}=\operatorname{tr}(\mathbf{C})$. Here, $\mathbf{F}=\mathbf{G r a d}(\boldsymbol{\varphi})$ and $\mathbf{C}=\mathbf{F}^{T} \mathbf{F}$ are the deformation gradient and right Cauchy-Green deformation tensor, respectively. The impact of the regularization parameter and the mechanical model utilized is further discussed in [11,15]. Following the general FE procedure, the variational form of Equation (1) is obtained by derivation, and then linearized and discretized using the standard continuous Lagrange elements. More details on the formulation, solution procedure and in vivo validation can be found in $[11,16]$.

Synthetic Image Generation and Method Implementation. Figure 1 shows the pipeline for the validation process. First, synthetic 3D-tagged images are generated from a reference biomechanical model of the LV [17, 18], combining a passive visco-hyperelastic behavior with a micro-macro model of the active muscle contraction [18], and coupled to a simplified cardiovascular circulation model [17]. FE resolution of the model provides the displacement field (from which one can compute any deformation metric, e.g., strain, strain rate, etc.) as well as the deformed configuration of LV throughout the whole cardiac cycle (Figure 1.a).

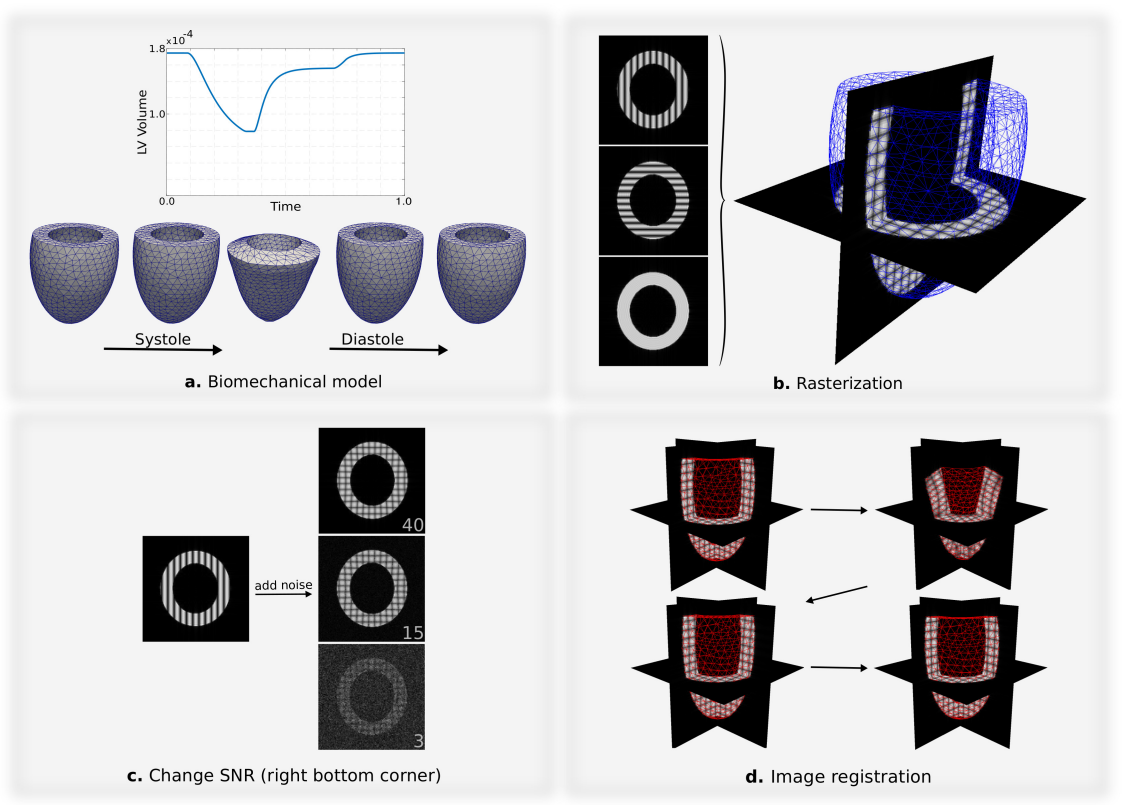

Fig. 1: (a) Reference biomechanical LV model simulating the full cardiac cycle [18]. (b) Synthetic image generation using the model shown in (a). Three different image stacks representing tag lines in each direction are generated and combined to have the 3D image (right). (c) Changing SNR by adding noise in image space. (d) Image registration showing the tagged images superimposed with the warped mesh for different time frames. 
The LV model is further utilized to generate synthetic 3D-tagged images through rasterization (Figure 1.b). For this purpose, image voxels lying inside the elements of the LV mesh are determined by rasterization [19] for each time frame and assigned the intensity $I_{0}$ for the reference configuration, which is a function of spatial coordinates $X_{0}, X_{1}$, and $X_{2}$ and the tagging period $\mathrm{s}$

$$
I_{0}(\mathbf{X})=\sqrt[3]{\left|\sin \left(\frac{\pi X_{1}}{s}\right)\right| \cdot\left|\sin \left(\frac{\pi X_{2}}{s}\right)\right| \cdot\left|\sin \left(\frac{\pi X_{3}}{s}\right)\right|}
$$

Images with resolution $0.5 \mathrm{~mm}$ and tag distance $7 \mathrm{~mm}$ are generated and then converted into $1 \mathrm{~mm}$ resolution using zero-filling technique in $\mathrm{k}$-space. These images are defined as reference dataset having infinite SNR, and are utilized for 3D validation of the FE-based image registration technique. By adding different levels of Gaussian noise with zero mean in the image space (Figure 1.c), images with eight different SNRs are generated to demonstrate the performance of the regularization technique in case of noise. Lastly, image registration is performed (Figure 1.d). For validation purposes, we computed normalized root mean square error (RMSE) in displacement field given by equation

$$
\mathrm{err}=\sqrt{\frac{\frac{1}{N_{t}} \frac{1}{N_{n}} \sum_{1}^{N_{t}} \sum_{1}^{N_{n}}\left\|\mathbf{u}^{n, t}-\mathbf{u}_{\mathrm{ref}}^{n, t}\right\|^{2}}{\left(\max \left\|\mathbf{u}_{\mathrm{ref}}\right\|\right)^{2}}},
$$

where $\mathbf{u}^{n, t}$ and $\mathbf{u}_{\mathrm{ref}}^{n, t}$ are the displacement vectors at node $\mathrm{n}$ and time $\mathrm{t}$, for the registered case and ground truth, respectively. Moreover, $N_{n}$ and $N_{t}$ are the total number of nodes and time frames. The method implementation is freely available at https://gitlab.inria.fr/mgenet/dolfin_dic as a python library implemented mainly in FEniCS [20] \& VTK libraries.

\section{Results \& Discussion}
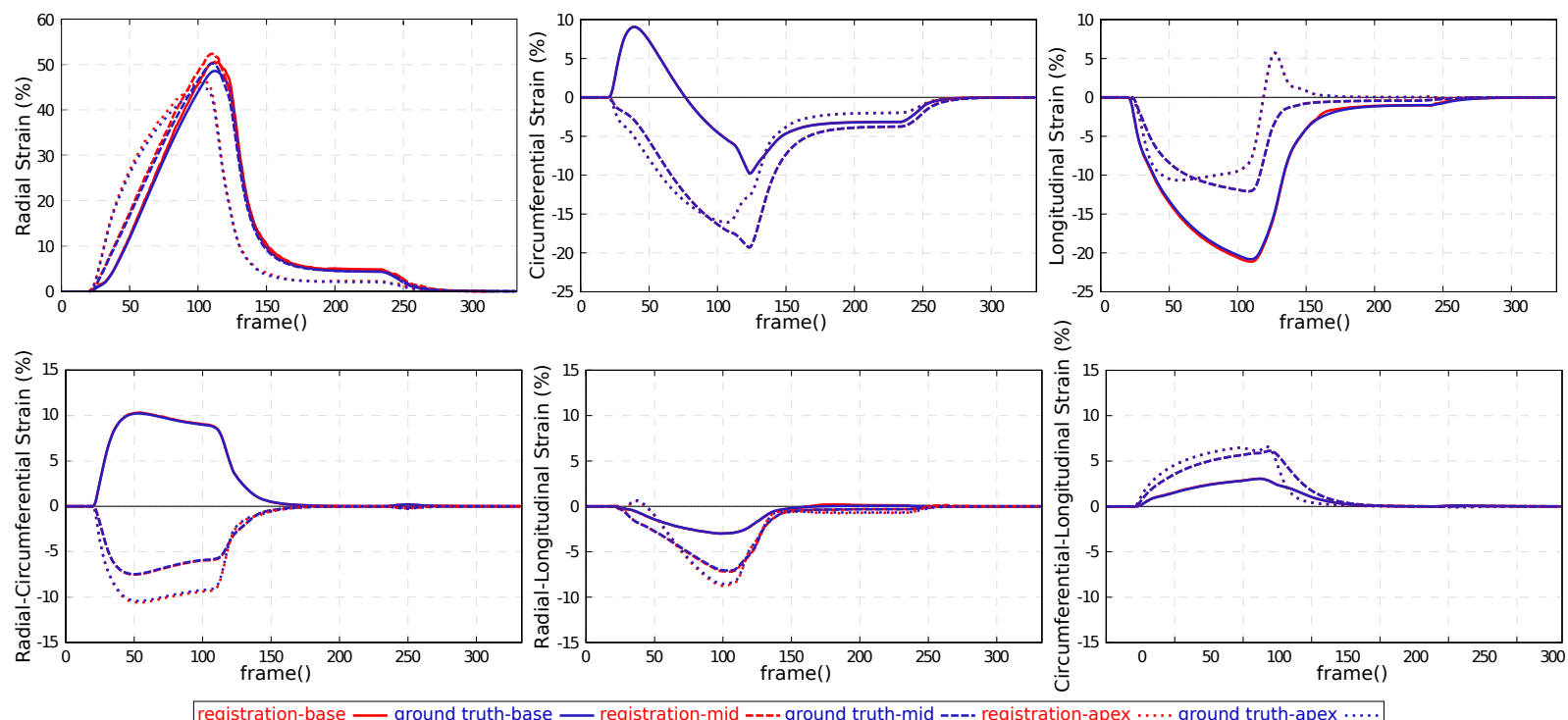

Fig. 2: Temporal variation of Green-Lagrange strain components for the mechanical model (blue curves) and the image registration (red curves). Strains are averaged over basal, mid-ventricular and apical regions of LV, represented by different line types.

First, FE-based image registration method is performed on the reference image set and Green-Lagrange strain components are computed for basal, mid-ventricular and apical regions of LV. Figure 2 shows the temporal variation of the regional strain components for the mechanical model and the registration by the blue and red curves, respectively. It can be concluded that the registration algorithm captures the deformation pattern of LV for the reference image set in terms of strain components. We further investigate the effect of image noise, commonly present in in vivo data. It has already been shown on 2D images that equilibrated warping can handle noisy images [11]. We run the registration algorithm for a range of regularization strength $\beta$ and plot RMSE given by Equation (6) for zero regularization $(\beta=0)$ and best performing case ( $\beta=$ best) in Figure 3.a. For infinite SNR, we have the smallest error in displacement field which is $0.4 \%$ regardless of the regularization strength utilized. As SNR decreases, registration problem requires regularization, which decreases error from $15.7 \%$ (for $\beta=0$ ) to $2.2 \%$ (for best $\beta$ ) for $\mathrm{SNR}=3$. Similar trend is found in Figure 3(b-d) in terms of the individual strain components. For images with $\mathrm{SNR} \geq 10$, zero regularization captures the ground truth strain components, while regularization helps significantly for lower SNR. 

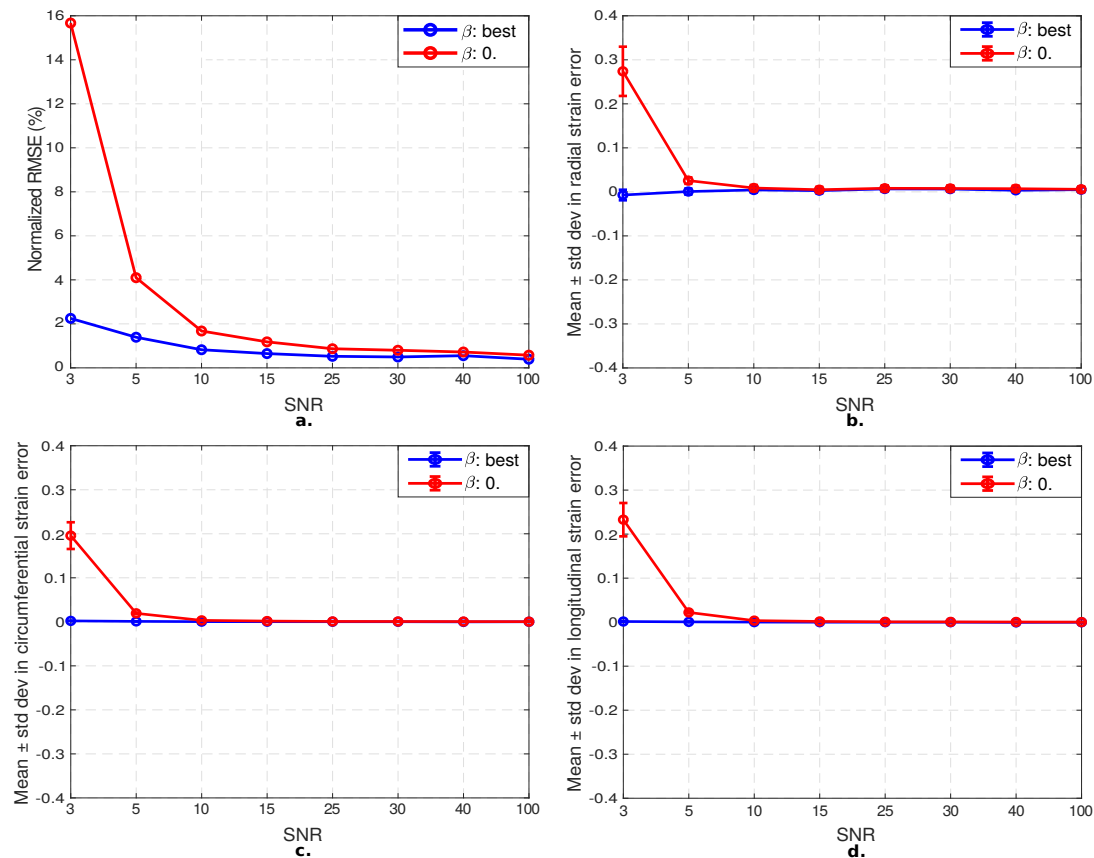

Fig. 3: Analysis results on images with resolution $1 \mathrm{~mm}$ and tag distance $7 \mathrm{~mm}$ as a function of SNR. (a) Normalized RMSE in displacement field for $\beta=0$ (red curve) and $\beta=$ best (blue curve). For $\mathrm{SNR} \leq 10$, regularization helps decreasing the registration error significantly. (b-d) Mean \pm standard deviation in component-wise strain error as a function of SNR which is independent of $\beta$ for SNR $\geq 10$.

\section{Concluding Remarks}

In this contribution, we have validated the recently proposed finite element image registration tool [11] on synthetic data. Equilibrated warping has been demonstrated to perform well to register the volumetric images of the deforming LV mesh, and the error in the generated displacement fields is shown to be negligible for large deformation mechanics. It has been further shown that the equilibrium gap constraint helps regularizing the registration problem even for very low SNR.

\section{References}

[1] H. Wang and A. A. Amini, IEEE Transactions on Medical Imaging 31(2), 487-503 (2012).

[2] T. P. Abraham and R. A. Nishimura, Journal of the American College of Cardiology 37(3), 731-734 (2001).

[3] J. D. Collins, Radiologic Clinics of North America 53(2), 369-395 (2015).

[4] A. Scatteia, A. Baritussio, and C. Bucciarelli-Ducci, Heart Failure Reviews 22(4), 465-476 (2017).

[5] E. A. Zerhouni, D. M. Parish, W. J. Rogers, A. Yang, and E. P. Shapiro, Radiology 169(1), 59-63 (1988).

[6] A. A. Young, C. M. Kramer, V. A. Ferrari, L. Axel, and N. Reichek, Circulation 90(2), 854-867 (1994).

[7] J. Bogaert and F. E. Rademakers, American Journal of Physiology. Heart and Circulatory Physiology 280(2), H610-620 (2001).

[8] A. W. Makram, A. M. Khalifa, H. El-Rewaidy, A. S. Fahmy, and E. S. H. Ibrahim, Japanese Journal of Radiology 34(2), 158-165 (2016).

[9] C. Tobon-Gomez, M. De Craene, K. McLeod, L. Tautz, W. Shi, A. Hennemuth, A. Prakosa, H. Wang, G. Carr-White, S. Kapetanakis, A. Lutz, V. Rasche, T. Schaeffter, C. Butakoff, O. Friman, T. Mansi, M. Sermesant, X. Zhuang, S. Ourselin, H. O. Peitgen, X. Pennec, R. Razavi, D. Rueckert, A. F. Frangi, and K. S. Rhode, Medical Image Analysis 17(6), 632-648 (2013).

[10] R. D. Rabbitt, J. A. Weiss, G. E. Christensen, and M. I. Miller, in: Vision Geometry IV (International Society for Optics and Photonics, 1995), pp. 252-266.

[11] M. Genet, C. T. Stoeck, C. von Deuster, L. C. Lee, and S. Kozerke, Medical Image Analysis 50, 1-22 (2018).

[12] A. Imperiale, R. Chabiniok, P. Moireau, and D. Chapelle, in: Functional Imaging and Modeling of the Heart, edited by D. N. Metaxas and L. Axel. Lecture Notes in Computer Science (Springer Berlin Heidelberg, 2011), pp. 409-417.

[13] D. Claire, F. Hild, and S. Roux, International Journal for Numerical Methods in Engineering 61(2), 189-208 (2004).

[14] P. Ciarlet and G. Geymonat, C. R. Acad. Sci. Paris Sér. II 295, 423-426 (1982).

[15] M. Genet, C. T. Stoeck, C. von Deuster, L. C. Lee, J. Guccione, and S. Kozerke, in: ISMRM 24rd Annual Meeting and Exhibition, (Singapore, May 2016).

[16] L.C. Lee and M. Genet in: Functional Imaging and Modeling of the Heart, edited by Y. Coudière, V. Ozenne, E. Vigmond and N. Zemzemi. Lecture Notes in Computer Science (Springer International Publishing, Cham, 2019), pp. 334-341.

[17] J. Sainte-Marie, D. Chapelle, R. Cimrman, and M. Sorine, Computers \& Structures 84(28), 1743-1759 (2006).

[18] D. Chapelle, P. L. Tallec, P. Moireau, and M. Sorine, International Journal for Multiscale Computational Engineering 10(2), 189-211 (2012).

[19] M. Sermesant, C. Forest, X. Pennec, H. Delingette, and N. Ayache, Medical Image Analysis 7(4), 475-488 (2003).

[20] M. Alnæs, J. Blechta, J. Hake, A. Johansson, B. Kehlet, A. Logg, C. Richardson, J. Ring, M. E. Rognes, and G. N. Wells, Archive of Numerical Software Vol 3 (2015). 